Ann. Zootech., I977, 26 (1), 69-8I.

\title{
Évolution de la composition chimique de jeunes agneaux mâles jusqu'à l'âge de 16 semaines
}

\author{
J. ROBELIN, M. THERIEZ *, M. ARNAL ** et M. FERRARA ** \\ Laboratoire de la Production de Viande, \\ * Station de Recherches sur l'Élevage des Ruminants, \\ ** Laboratoire d'Étude du Métabolisme Azoté, \\ Centre de Recherches de Clermont-Ferrand, I.N.R.A., \\ Theix, Saint-Genès-Champanelle 63IIo Beaumont (France)
}

\begin{abstract}
Résumé
L'évolution de la composition chimique du corps entier des agneaux mâles a été étudiée grâce à l'abattage de 4 groupes de 5 animaux âgés de I, 5, Io et 16 semaines et dont le poids vif était égal en moyenne à 4,$8 ; 12,4 ; 23,5$ et $32,9 \mathrm{~kg}$ respectivement.

Sur l'ensemble de la période étudiée, le pourcentage d’eau dans le corps vide a diminué entre 73,7 et $64,5 \mathrm{p}$. Ioo tandis que le pourcentage de matières grasses augmentait entre 6,3 et I 4, I p. Ioo, et la valeur calorifique entre I 428 et $2230 \mathrm{kcal} / \mathrm{kg}$. Le pourcentage de protéines est resté pratiquement constant et voisin de 16,5 p. Ioo.

La période de croissance étudiée a été scindée en 3 phases : I à 5 semaines (alimentation lactée); 5 à Io semaines (sevrage); Io à I6 semaines (engraissement). L'augmentation relative de contenu en matières grasses a tout d'abord été rapide (coefficient d'allométrie $b=1,69$ ). Elle a été ralentie au cours de la seconde période $(b=1,22)$, puis à nouveau rapide au cours de la dernière période $(b=1,4 \mathrm{I})$. La rétention protéique a subi les mêmes fluctuations, mais en sens inverse L'évolution de la composition du gain de poids vif en matières grasses, protéines et énergie a été discutée en fonction des variations dans l'alimentation des animaux et de leur état physiologique (puberté).
\end{abstract}

\section{Introduction}

L'évolution du poids des organes et des composants tissulaires (os, muscles, dépôts adipeux) au cours de la croissance chez l'agneau est relativement bien connue, grâce aux travaux récents, réalisés aux U.S.A. (LoHSE et al., I97r), en Nouvelle-Zélande (Fourie et al., I970; Kirton et al., I972) et en France (BeneVENT, I97I; BOCCARD et DumONT, I973). En revanche, les travaux sur l'évolution de la composition chimique ont été plus limités. Ils n'ont souvent concerné qu'une courte période de la vie, soit avant le sevrage (JAGUSH et al., 1970), soit pendant 
la phase d'engraissement (Rouse et al., I970; ANDREws et Orskov, I970; KIRTON et al., I974). Enfin, ces études ont été réalisées le plus souvent avec des animaux castrés, dont le développement doit être très différent de ceux des mâles entiers à croissance rapide, utilisés en France pour la production de viande.

Cette étude a eu pour objet. de décrire l'évolution de la composition chimique des agneaux mâles entiers, jusqu'à l'âge de 16 semaines, par la méthode des abattages.

\section{Matériel et méthodes}

\section{Animaux}

Quatre groupes de 5 agneaux mâles, choisis sur la base du poids vif, ont été abattus aux âges de I, 5, Io ou I6 semaines; ces stades d'abattage ont été choisis de façon à décrire l'évolution de la composition chimique des animaux, avant et après le sevrage (respectivement entre I et 5 semaines, et entre Io et I6 semaines), et à subdiviser la période de croissance en phases de durées comparables. Le dernier stade ( 16 semaines) correspond pratiquement au stade d'abattage commercial.

Les animaux ont été choisis sur la base du poids et de l'âge, parmi une popu-

\section{TABLEAU I}

Quantités d'aliments ingérées par jour par les agneaux mesurées la semaine précédant l'abattage

Daily feed intake in the lambs measured the week before slaughter

\begin{tabular}{|c|c|c|c|}
\hline \multirow{2}{*}{$\begin{array}{c}\text { Période de mesures } \\
\text { (Period of measurement) }\end{array}$} & \multirow{2}{*}{$\begin{array}{l}\text { Aliments } \\
(\text { Feed })\end{array}$} & \multicolumn{2}{|c|}{$\begin{array}{l}\text { Matière sèche } \\
(\text { Dry matter })\end{array}$} \\
\hline & & $\mathrm{g} / \mathrm{j}( \pm \sigma)$ & $\mathrm{g} / \mathrm{kgP} \quad 0.75 / \mathrm{j}$ \\
\hline $\begin{array}{l}\text { Semaine I } \\
(\text { Week } I)\end{array}$ & $\begin{array}{l}\text { Lait } \\
(\text { Milk })\end{array}$ & $219 \quad(82)$ & 78,2 \\
\hline $\begin{array}{l}\text { Semaine } 4 \\
(\text { Week } 4)\end{array}$ & $\begin{array}{c}\text { Lait } \\
\text { (Milk) } \\
\text { Concentré } \\
\text { (Concentrate feed) }\end{array}$ & $\begin{aligned} 4 \mathrm{I}_{4} & (7) \\
\mathrm{I} 2 & (\mathrm{II})\end{aligned}$ & $\begin{array}{r}66,6 \\
1,9\end{array}$ \\
\hline $\begin{array}{l}\text { Semaine } 9 \\
(\text { Week } 9)\end{array}$ & $\begin{array}{c}\text { Concentré } \\
\text { (Concentrate feed) } \\
\text { Foin } \\
(\text { Hay })\end{array}$ & $\begin{array}{l}944(\mathrm{r} 64) \\
\text { 100 (59) }\end{array}$ & $\begin{array}{l}9 \mathrm{I}, 3 \\
10,0\end{array}$ \\
\hline $\begin{array}{l}\text { Semaine } 15 \\
\left(\begin{array}{ll}\text { Week } & \text { 5 }\end{array}\right)\end{array}$ & $\begin{array}{c}\text { Concentré } \\
\text { (Concentrate feed) } \\
\text { Foin } \\
(\text { Hay })\end{array}$ & $\begin{array}{r}1105(171) \\
129 \quad(71)\end{array}$ & $\begin{array}{r}81,9 \\
9,6\end{array}$ \\
\hline
\end{tabular}


lation de $5_{50}$ agneaux mâles entiers, nés à la même époque, issus de pères appartenant à la race Berrichonne du Cher et de mères croisées Romanov $\times$ Limousines. Les agneaux ont été séparés de leur mère à la naissance et élevés en lot. Ils ont reçu jusqu'à l'âge de 5 semaines, un aliment d'allaitement comportant 25,8 p. Ioo de matières azotées totales et $25 \mathrm{p}$. Ioo de matières grasses. Ils ont été sevrés progressivement au cours de la sixième semaine, et ont reçu un aliment concentré comportant $\mathrm{I} 7,8 \mathrm{p}$. Ioo de matières azotées totales et du foin de luzerne. Les quantités moyennes d'aliment ingérées, au cours de la semaine précédant l'abattage, sont rapportées sur le tableau $\mathrm{I}$. Les vitesses de croissance des animaux ont été comprises entre 240 et $280 \mathrm{~g} / \mathrm{j}$.

\section{Abattage des animaux et mesure de la composition chimique}

Après avoir été pesés, les animaux ont été abattus et saignés. Le sang et l'ensemble du contenu digestif ont été pesés et échantillonnés en vue de la mesure de la teneur en eau. Ensuite, le reste de l'animal (non tondu) a été découpé (sans considération anatomique) en morceaux, d'un poids voisin de $500 \mathrm{~g}$, qui ont été congelés dans l'azote liquide et broyés ensemble dans un broyeur à hélice (Stephan). Les pertes en eau, au cours de ces manipulations, ont été en moyenne inférieures à I p. Ioo du poids vif vide. De l'ensemble du broyat de chaque animal, ont été prélevés 18 échantillons (pesant $200 \mathrm{~g}$ environ) qui ont été lyophilisés pour déterminer la teneur en eau. L'intervalle de confiance $(P<0, \mathbf{I} 0)$ de la moyenne de ces I8 mesures a été égal à \pm 0, ,I $p$. Ioo du produit frais.

La teneur en matières grasses du mélange des I 8 échantillons (et des échantillons de sang) a été mesurée sur 8 prises d'essai par une méthode dérivée de celle de Folch (triple extraction par un mélange de 2 volumes de chloroforme, I volume de méthanol, et purification sur filtre de sulfate de sodium anhydre). Ramené en pourcentage du produit frais, l'intervalle de confiance $(\mathrm{P}<0, \mathrm{IO})$ de la moyenne des 8 déterminations a été égal à $0,08 \mathrm{p}$. Ioo. La teneur en cendres a été mesurée par passage des échantillons au four à $600^{\circ}$ pendant 8 heures. L'erreur sur cette mesure a été égale à $0, \mathbf{I} 3$ p. Ioo du produit frais.

La teneur en protéines a été mesurée par différence (RoBELIN et GEAY, I976). L'erreur sur cette détermination (somme des autres erreurs) a été voisine de 0,30 p. roo, ce qui est relativement faible.

Enfin, la valeur calorifique a été mesurée (moyenne de 6 résultats), par calorimétrie adiabatique (erreur égale à $36 \mathrm{cal} / \mathrm{g}$ de la matière fraîche).

\section{Analyse des résultats}

Nous avons calculé la composition du gain de poids des animaux, en considérant que la composition des agneaux, à chaque stade d'abattage, était représentative de celle des agneaux de la population dont ils étaient issus.

Nous avons également relié le poids des différents constituants chimiques étudiés $(\mathrm{Y})$ à celui du corps vide $\left(\mathrm{X}_{1}\right)$ par l'équation logarithmique $(\log \mathrm{Y}=b$ 
$\log \mathrm{X}+a)$ de Huxley, et nous avons comparé les pentes $b$ de ces équations correspondant aux différentes périodes étudiées ( $\mathrm{I}$ à 5,5 à Io et Io à I 6 semaines) par analyse de covariance. Nous avons également comparé, par la même méthode, les différences entre périodes dans les relations entre le poids vif vide $\left(\mathrm{X}_{1}\right)$, le poids d'eau du corps vide $\left(\mathrm{X}_{2}\right)$ et le poids des autres constituants chimiques $(\mathrm{Y})$ :

$$
\log (\mathrm{Y})=b_{1} \log \left(\mathrm{X}_{1}\right)+b_{2} \log \left(\mathrm{X}_{2}\right)+b_{0}
$$

Cette transformation logarithmique permet de se rapprocher des conditions de normalité auxquelles font appel les tests statistiques, en rendant la variabilité des différents poids moins dépendante des poids eux-mêmes. De plus, l'écart type Syx de ces équations logarithmiques possède une propriété intéressante. En effet, $\mathrm{SD}=\mathrm{IOO} \times \mathrm{IO}^{\mathrm{gyx}}$ représente la variation résiduelle de $\mathrm{Y}$ exprimée en pourcentage de $Y$, que nous nommerons coefficient de variation résiduelle (C.V.R.).

\section{Résultats}

\section{Croissance globale des agneaux}

Le gain de poids journalier a été égal à $233 \mathrm{~g}$ en moyenne $( \pm 66 \mathrm{~g})$ au cours de la première semaine. Il a été plus élevé, et pratiquement constant entre les âges de $I$ et ro semaines $(280 \mathrm{~g} / \mathrm{j})$, puis il a diminué au cours de la période d'engraissement, entre les âges de Io et 16 semaines $(240 \pm 23 \mathrm{~g} / \mathrm{j}$ ) (tabl. 2). Cette vitesse de croissance n'est pas très élevée pour ce type d'animaux, surtout en période de finition entre Io et $\mathbf{I} 6$ semaines, où l'on peut couramment atteindre 300 à $350 \mathrm{~g} / \mathrm{j}$.

TABLEAU 2

Croissance globale des agneaux

Overall growth of the lambs

\begin{tabular}{|c|c|c|c|}
\hline $\begin{array}{c}\text { Nombre } \\
\text { d'animaux } \\
\text { (Number of animals) }\end{array}$ & $\begin{array}{c}\text { Age moyen } \\
\text { (Mean age) } \\
\text { semaines } \\
(\text { Weeks })\end{array}$ & $\begin{array}{l}\text { Poids vif } \\
(\text { Live weight }) \\
(\mathrm{kg} \pm \sigma)\end{array}$ & $\begin{array}{c}\text { Gain de poids vif } \\
\text { (Live weight gain) } \\
\{\mathrm{g} / \mathrm{j} \pm \sigma)\end{array}$ \\
\hline 20 & o & $3,08 \pm 0,4^{2}$ & \\
\hline 20 & $\mathrm{r}$ & $4,8 \mathrm{I} \pm 0,54$ & $233 \pm 66$ \\
\hline I5 & 5 & $12,39 \pm 0,92$ & $278 \pm 26$ \\
\hline Io & IO & $23,52 \pm 1,94$ & $279 \pm 54$ \\
\hline 5 & 16 & $32,94 \pm x, 48$ & $24^{\circ} \pm 23$ \\
\hline
\end{tabular}

Evolution de la composition $d u$ corps vide

Tandis que le poids vif vide passait de 4,90 à $28,77 \mathrm{~kg}$ entre les âges de I et I6 semaines (tabl. 3), le pourcentage d'eau, dans le corps vide, a diminué de 73,9 à 64,5 ; le pourcentage de matières grasses et la valeur calorifique ont augmenté 
TABLEAU 3

Age, poids et composition chimique des agneaux à chaque stade d'abattage

Age, weight and chemical composition of lambs at each slaughter.

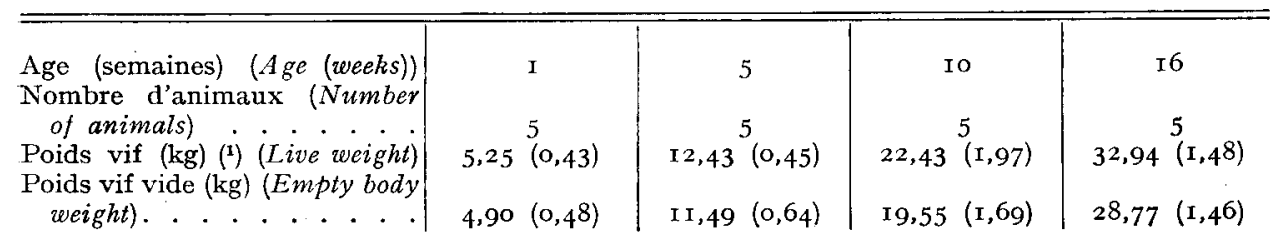

Répartition des constituants du corps vide (sans contenu digestif) (Distribution of empty body constituents (zerithout gut content))

\begin{tabular}{|c|c|c|c|c|}
\hline $\begin{array}{l}\text { Eau du corps vide }(\mathrm{kg}) \text { (Empty } \\
\text { body water }) .\end{array}$ & $3,62(0,36)$ & $7,87 \quad(0,36)$ & I $2,96(0,98)$ & $\mathrm{I} 8,57(\mathrm{I}, \mathrm{II})$ \\
\hline Matières grasses $(\mathrm{kg})$ (Fat) & $0,3 \mathbf{I}(0,05)$ & $\mathrm{I}, 33(0,16)$ & $2,44(0,57)$ & $4,08 \quad(0,29)$ \\
\hline Protéines $(\mathrm{kg})$ (Protein). & $0,80(0,07)$ & $1,92(0,13)$ & $3,42(0,25)$ & $4.99(0,26)$ \\
\hline Cendres $(\mathrm{kg})$ (Ashes) ... & $0,17(0,02)$ & $0,37(0,05)$ & $0,73(0,04)$ & I, I3 $(0,04)$ \\
\hline Énergie (Mcal) (Energy). & $7,00(0,8 \mathrm{r})$ & $22,83(2,05)$ & $4^{0,37}(6,84)$ & $64,15 \quad(1,88)$ \\
\hline
\end{tabular}

Composition moyenne du corps vide (p. Ioo) (Mean composition of empty body)

\begin{tabular}{|c|c|c|c|c|}
\hline Eau (Water) . . . & 73,87 & 68,19 & 66,29 & 64,54 \\
\hline Matières grasses (Fat). & 6,32 & II, 57 & I 2,48 & $I_{4}, 18$ \\
\hline Protéines (Protein) . & 16,32 & $16,7 \mathrm{I}$ & 17,49 & $\mathrm{r} 7,34$ \\
\hline Cendres (Ashes) . . . & 3.46 & 3,22 & 3,73 & 3,92 \\
\hline Énergie (Cal/g) (Energy). & I $4^{28}$ & I 987 & 2065 & $223^{\circ}$ \\
\hline
\end{tabular}

(1) Les valeurs indiquées entre parenthèses sont les écarts types. (Values between brackets represent standard deviations.)

respectivement de 6,3 à I4, I et de I 428 à $2230 \mathrm{kcal} / \mathrm{kg}$. Le pourcentage de protéines a augmenté très faiblement de $I 6,3$ à I7,3. Le pourcentage d'eau dans la masse délipidée (poids vif vide - matières grasses) a diminué rapidement entre les âges de I à ro semaines (de 78,8 à 75,7 ), et plus lentement ensuite (de 75,7 à $75,2)$.

Cette évolution de la composition chimique du corps vide n'a pas été continue entre les âges de $I$ et I6 semaines. Ainsi, l'augmentation relative du contenu en matières grasses, par rapport au poids vif vide (tabl. 4), a été significativement plus élevée pendant la première période $(b=\mathrm{I}, 69)$ que pendant les périodes suivantes $(b=\mathrm{I}, 22$ et $\mathrm{I}, 4 \mathrm{I})$. De même, l'augmentation relative de la valeur calorifique a été plus forte pendant la première période $(I, 35$ contre I,II et I,26). L'augmentation relative du contenu en cendres a été au contraire plus faible au cours de cette première phase $(0,82$ contre $\mathrm{I}, \mathrm{I} 5$ et $\mathrm{I}, \mathrm{OO})$. Enfin, l'augmentation relative du contenu en eau ou en protéines n'a pas évolué significativement (respectivement $0,93,0,94,0,93$ et 0,$98 ; \mathrm{I}, 0 \mathrm{r}, 0,95)$. Il est pourtant intéressant de remarquer que la croissance des protéines est passée par un maximum $(b=\mathrm{I}, \mathrm{OI})$ au cours de la $2^{\mathrm{e}}$ période (fig. $\mathrm{r}$ ). 
TABLEAU 4

Coefficients d'allométrie des différents constituants chimiques du corps vide par rapport au poids vif vide, chez les agneaux entre I et I6 semaines Allometric coefficients of the different chemical constituents of the empty body in velation to empty body weight in lambs between $x$ and $x 6$ weeks

\begin{tabular}{|c|c|c|c|}
\hline & Période I & Période 2 & Période 3 \\
\hline $\begin{array}{l}\text { Age des animaux (semaines) (Age of ani- } \\
\quad \text { mals (weeks)) } \\
\text { Poids vif vide (kg) (Empty body weight). }\end{array}$ & $\begin{array}{c}I-5 \\
4,3-I 2,0\end{array}$ & $\begin{array}{c}\text { 5-10 } \\
10,8-2 \mathrm{I}, 5\end{array}$ & $\begin{array}{c}\text { I0-16 } \\
17,8-31,2\end{array}$ \\
\hline 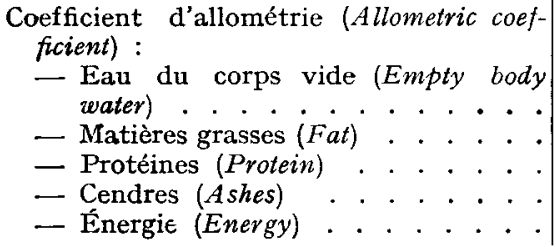 & $\begin{array}{l}0,93^{a} \\
\mathrm{I}, 69^{a} \\
\mathrm{0}, 98^{a} \\
\mathrm{o}, 82^{a} \\
\mathrm{I}, 35^{a}\end{array}$ & $\begin{array}{l}0,94^{a} \\
\mathrm{I}, 22^{b} \\
\mathrm{I}, 01{ }^{a} \\
\mathrm{I}, \mathrm{I} 5^{b} \\
\mathrm{I}, \mathrm{II} \mathrm{I}^{b}\end{array}$ & $\begin{array}{l}0,93^{a} \\
1,4^{a b} \\
0,95^{a} \\
1,00^{a b} \\
1,26^{a b}\end{array}$ \\
\hline
\end{tabular}

Les coefficients relatifs à chaque période ont été comparés entre eux par analyse de covariance (test sur les différences de pentes). Les résultats non significativement différents (au niveau o,or) sur une même ligne sont accompagnés de la même lettre $a$ ou $b$. (Relative coefficients at each period were compared by covariance analysis (test on slope differences). Not significantly different data (at the o.or level) on the same row are accompanied by the same letter a or b.)

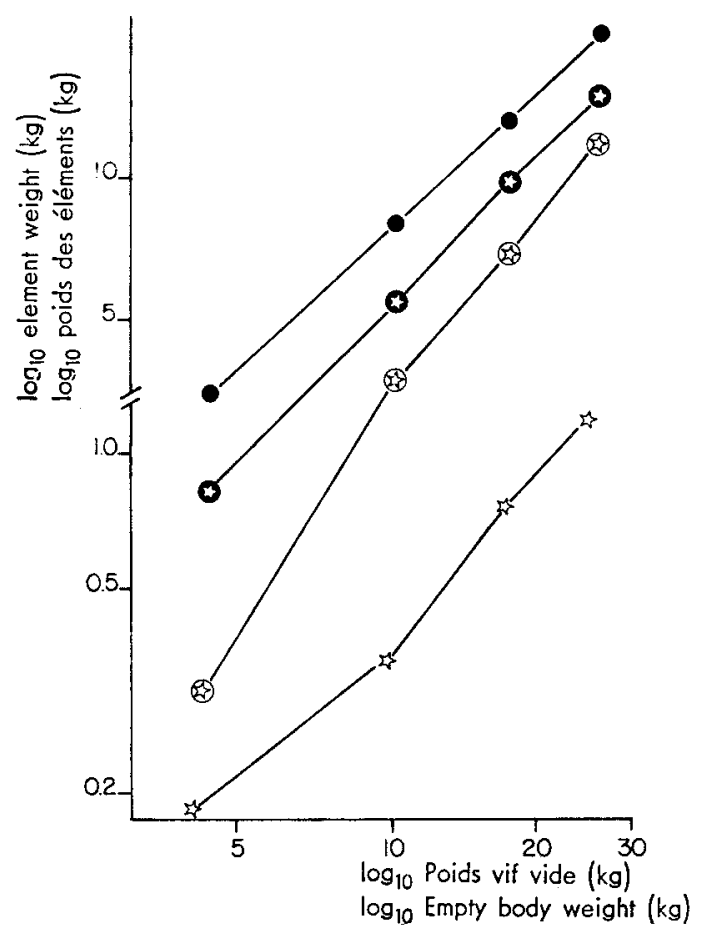

FIG. I. - Evolution du poids des matières grasses des protéines 1 de l'eau $\bullet$, et des minéraux chez les agneaux entre 4 et $29 \mathrm{~kg}$ de poids wif vide (Changes in the weight of fat, protein $\bullet$, water , minerals in lambs between 4 and $29 \mathrm{~kg}$ empty body weight.) 


\section{Composition $d u$ gain de poids vif}

Au cours des deux premières périodes (I à 5 et 5 à ro semaines), la croissance globale est restée constante ( $280 \mathrm{~g} / \mathrm{j}$ en moyenne), alors que la croissance du corps vide a diminué (de 255 à $225 \mathrm{~g} / \mathrm{j}$ ) en raison de l'augmentation du contenu digestif (période du sevrage) (tabl. 5). Ces modifications ont été accompagnées d'une réduction de la quantité d'énergie fixée par jour (de 6r 2 à $490 \mathrm{kcal}$ ).

\section{TABLEAU 5}

Croissance des différents constituants du corps entier chez l'agneau entre $I$ et I6 semaines

Growth of the different whole body constituents in lambs between $I$ and $I 6$ weeks

\begin{tabular}{|c|c|c|c|}
\hline $\begin{array}{c}\text { Périodes } \\
\text { (Periods) } \\
\text { (semaines) } \\
\text { (weeks) }\end{array}$ & I à 5 & 5 à Io & Io à 16 \\
\hline 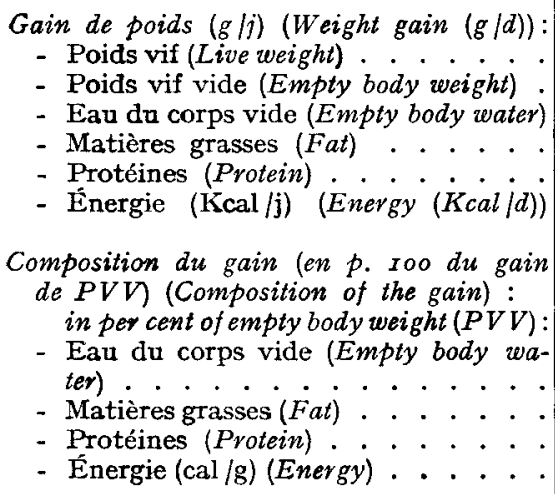 & $\begin{array}{r}278 \\
255 \\
164 \\
39 \\
43 \\
612\end{array}$ & $\begin{array}{r}279 \\
225 \\
142 \\
31 \\
42 \\
490\end{array}$ & $\begin{array}{r}240 \\
210 \\
\text { I } 28 \\
37 \\
36 \\
543\end{array}$ \\
\hline
\end{tabular}

Au cours de la $3^{\mathbf{e}}$ période ( $\mathrm{ro}$ à $\mathbf{I} 6$ semaines), la croissance du corps vide a été légèrement plus faible que précédemment (2I0 $\mathrm{g} / \mathrm{j})$. Cette diminution a été accompagnée d'une très forte réduction de la rétention des protéines (de 42 à $36 \mathrm{~g} / \mathrm{j}$ ) et d'une augmentation de l'accumulation de matières grasses (3I à $37 \mathrm{~g} / \mathrm{j}$ ) qui s'est traduite par un accroissement de la quantité d'énergie fixée par jour (de 490 à $543 \mathrm{kcal})$.

Compte tenu de cette évolution, le kilogramme de gain de poids vif vide a comporté, au cours des trois périodes successives, 645, 63I et $608 \mathrm{~g}$ d'eau; I55. I38 et $I_{7} 8 \mathrm{~g}$ de matières grasses; I70, I86 et I70 $\mathrm{g}$ de protéines et 2402,2 I 76 et $2579 \mathrm{kcal}$ (tabl. 5). 
Relations entre les différents constituants chimiques du corps vide

Les variations du poids vif vide ont expliqué 99 p. Ioo des variations de poids des constituants chimiques (tabl. 6); c'est le poids de matières grasses qui a eu la plus forte variabilité résiduelle (coefficient de variation $\mathrm{SD}_{1}=12,3 \mathrm{p}$. IOo), mais il a été par ailleurs, comme la valeur calorifique, lié significativement au poids d'eau du corps vide. Ainsi, lorsque le poids vif vide et le poids d'eau du corps vide

\section{TABLEAU 6}

Analyse des relations entre le poids des différents constituants chimiques du corps vide $(Y)$, le poids vif vide (PVV) et le poids d'eau du corps vide (ECV) (1)

Analysis of velationships between the weight of empty body chemical constituents ( $Y$ ). empty body weight (PVV) and empty body water weight (ECV)

\begin{tabular}{|c|c|c|c|c|c|}
\hline \multirow[t]{2}{*}{$\begin{array}{l}\text { Variables dépendantes } \\
\text { (Dependent variables) }\end{array}$} & \multicolumn{2}{|c|}{$\begin{array}{l}\text { Variables indépendantes } \\
\text { (Independent variables) }\end{array}$} & \multirow{2}{*}{$\begin{array}{l}\text { Niveau } \\
\text { de signi- } \\
\text { fication } \\
\text { de E.C.V. } \\
\text { (Level of } \\
\text { significance } \\
\text { of E.C.V.) }\end{array}$} & \multicolumn{2}{|c|}{\begin{tabular}{|} 
Niveau de signification \\
des différences \\
entre périodes \\
(Level of significance \\
of differences \\
between periods)
\end{tabular}} \\
\hline & P.V.V. & $\begin{array}{l}\text { P.V.V. } \\
\text { et E.C.V. }\end{array}$ & & Y/P.V.V. & $\begin{array}{l}\text { Y/P.V.V. } \\
\text { et E.C.V. }\end{array}$ \\
\hline \multicolumn{6}{|l|}{ Eau (Water) : } \\
\hline $\left.\mathrm{R}^{2}{ }^{2}{ }^{2}\right) \ldots \ldots$ & 0,9995 & - & - & NS & - \\
\hline $\begin{array}{l}\left.\text { SD p. roo }{ }^{3}\right\rangle \\
\text { Matières grasses } \\
\left(F_{\text {Fat }}\right)\end{array}$ & $\mathrm{I}, 7$ & - & - & 一 & 一 \\
\hline $\mathrm{R}^{2} \ldots$ & 0,9892 & 0,9953 & $0,0 \mathrm{r}$ & 0,10 & 0, IO \\
\hline $\begin{array}{l}\text { SD p. roo } \\
\text { Protéines (Protein) }\end{array}$ & 12,3 & 6,9 & - & - & - \\
\hline $\begin{array}{c}\text { Proteines }(\text { Protein }): \\
\mathrm{R}^{2} . . . . . .\end{array}$ & 0,9984 & 0,0987 & NS & NS & - \\
\hline $\begin{array}{l}\text { SD p. roo : : . } \\
\text { Cendres (Ashes) : }\end{array}$ & 2,5 & 2,5 & - & - & - \\
\hline $\begin{array}{l}\text { Cendres (Ashes) : } \\
\mathrm{R}^{2} . \ldots \ldots . . . . .\end{array}$ & 0.0885 & O OOOT & & 005 & \\
\hline SD p. 10o . . . . . & 7,6 & 7,6 & No & $\underline{0,05}$ & 二 \\
\hline Energie (Energy) : & & & & & \\
\hline $\mathrm{R}^{\mathrm{a}} \ldots \ldots \ldots$ & 0,9962 & 0,9985 & 0,01 & 0,05 & 0,05 \\
\hline SD p. Iоo . . . . . & 5,9 & 2,9 & - & - & - \\
\hline
\end{tabular}

(1) Les résultats ont été ajustés à deux relations $(\mathrm{A}): \log (\mathrm{Y})=a_{1} \log (\mathrm{PVV})+a_{2}$ et $(\mathrm{B}): \log (\mathrm{Y})$ $=b_{1} \log (\mathrm{PVV})+b_{2} \log \mathrm{ECV}+b_{3}$ calculées sur les trois périodes ( $\mathrm{I}$ à 5 semaines, 5 à Io semaines et Io à I6 semaines). La signification de ECV a été testée sur la différence entre les carrés des coefficients de corrélation $\left(R^{2}\right)$ des relations (B) et (A). La signification des différences entre périodes dans les coefficients $a_{1}$, $b_{1}, b_{2}$ a été testée par analyse de covariance. (Results were adjusted to 2 relations $(A): \log (Y)=\mathbf{a}_{1} \log$ $(P V V)+\mathrm{a}_{2}$ and $(B): \log (Y)=\mathrm{b}_{1} \log (P V V)+\mathrm{b}_{2} \log E C V+\mathrm{b}_{3}$ calculated on the 3 periods ( $\mathrm{I}-5$ weeks, 5-IO weeks and ro-I6 weeks). Significance of ECV was tested on the difference between the correlation coefficient squares $\left(R^{2}\right)$ of the relations $(B)$ and $(A)$. Significance of intra-period differences in the coeficients $\mathbf{a}_{1}$, $\mathbf{b}_{1}, \mathbf{b}_{2}$ was tested by covariance analysis.) coefficient.)

$\left.{ }^{(}\right) R^{2}$ est le carré du coefficient de corrélation intra-période. $\left(R^{2}=\right.$ square of intra-period correlation

$\left({ }^{3}\right)$ SD p. roo représente l'écart type résiduel de $\mathrm{Y}$ exprimé en pourcentage de $\mathrm{Y}$. (SD per cent represents the residual standard deviation of $Y$ expressed in percent of $Y$.) 
étaient connus, la variabilité résiduelle $\left(\mathrm{SD}_{2}\right)$ du poids des matières grasses était égale à 6,9 p. Ioo seulement. Les poids des protéines et des minéraux n'ont pas été liés significativement au poids d'eau.

Un certain nombre de relations ont eu des coefficients significativement différents selon les périodes de croissance (tabl. 6) :

- Relation entre le poids des matières grasses et le poids vif vide.

- Relation entre le poids des cendres et le poids vif vide.

- Relation entre la valeur calorifique et le poids vif vide.

- Relation entre le poids des matières grasses, le poids vif vide et l'eau du corps vide.

\section{Discussion}

L'ensemble de ces résultats a mis en évidence des variations importantes dans la composition du gain de poids des agneaux au cours des 3 périodes que nous avons étudiées; cela n'est pas surprenant au regard des différences d'ordres physiologique et nutritionnel entre ces trois phases : les animaux sont passés d'une alimentation lactée à une période de sevrage, puis à une alimentation à base de foin et d'aliments concentrés. De plus, dans la période allant de 5 à ro semaines (I2 à $23 \mathrm{~kg}$ ), se situe l'apparition de la puberté.

La seconde période étudiée, période du sevrage, a surtout été marquée par une augmentation importante du poids du contenu digestif (de 7,I à I 2,7 p. IoO du poids vif), qui a entraîné une chute de la croissance pondérale réelle (croissance du corps vide). Cette augmentation du contenu digestif au cours du sevrage a été comparable à celle qu'ont observée MATHIEU et al. (résultats non publiés) sur les bovins. Elle correspond à une augmentation très importante à cette période de la croissance relative des estomacs (BENEVENT, I97I).

Cette période, comprise entre 5 et Io semaines, a été marquée également par une chute brutale de l'accumulation des matières grasses, comme l'ont déjà observé Benevent (I97I) et Searle et al. (I972). Molenat et al. (I97I) ont montré que les quantités d'aliments ingérées et le gain de poids vif diminuaient considérablement au cours de la première semaine de sevrage. Il est probable que c'est la diminution des quantités d'énergie ingérées (liée à la composition des aliments et à leur utilisation) qui a entraîné, sur l'ensemble de la période suivant le sevrage (5 à ro semaines), une réduction de la croissance du corps vide, modifiant surtout le dépôt des matières grasses. Il est également possible, comme le suggérait BENEVENT (I97I), que la réduction de la lipogénèse soit liée à la modification d'équilibres endocriniens, précédant l'apparition de la puberté. Cette diminution de l'adipogénèse au moment de la puberté a d'ailleurs été observée également chez les bovins, entre 250 et $300 \mathrm{~kg}$, par Almouist et al. (I97 I), et par Schultz et al. (r974). Le fait que le dépôt de protéines n'ait pas été modifié au cours de cette période, est probablement lié un rendement de fixation (protéines retenues/protéines synthétisées) particulièrement élevé à cet âge (ARNAL et al., I976).

Au cours de la troisième période (engraissement), la vitesse de dépôt des protéines a diminué, tandis que le dépôt de gras augmentait. Ce résultat est tout à fait conforme au schéma de HAmmond. Toutefois, la part des matières grasses dans 


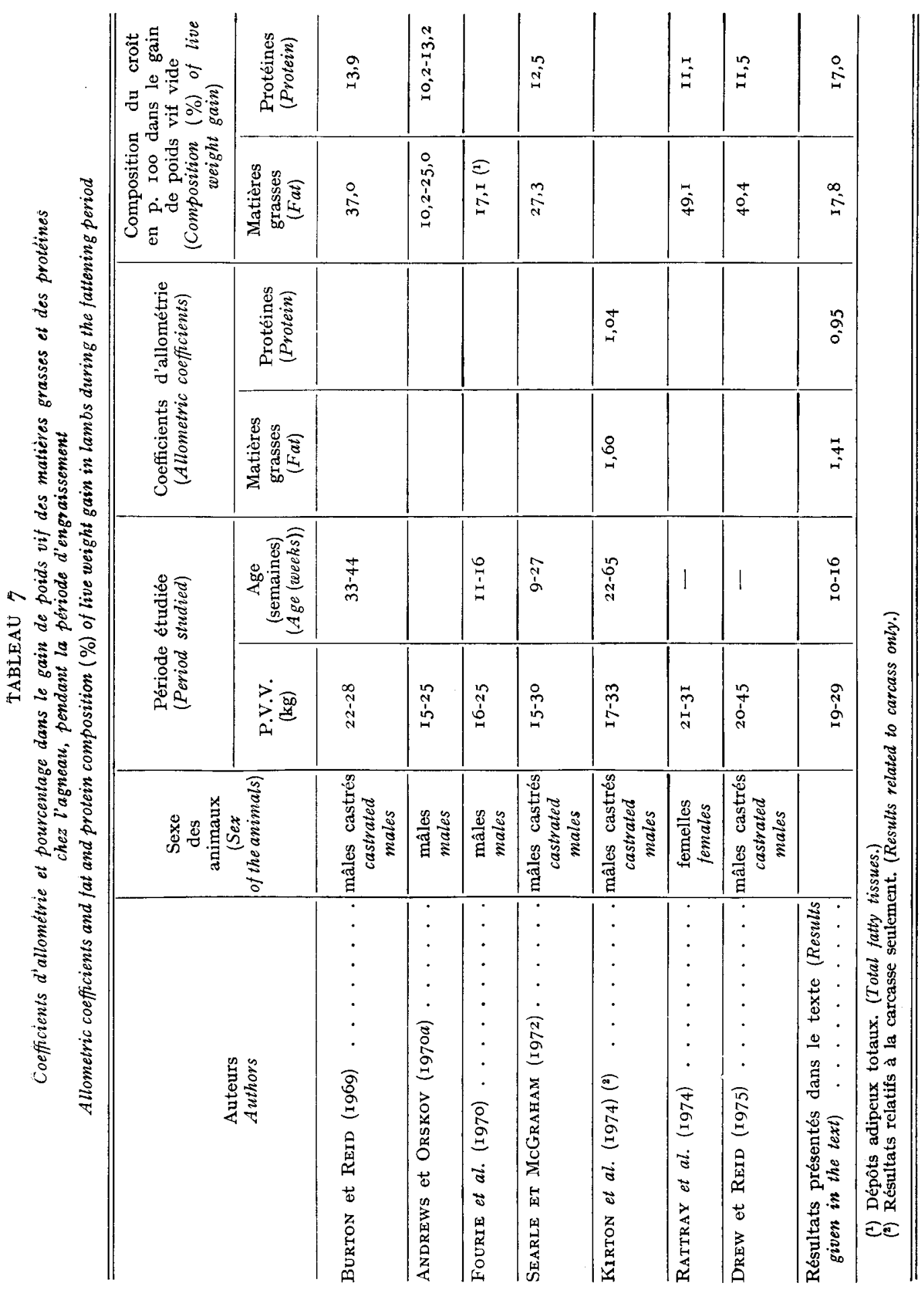


le gain de poids vif vide a été relativement faible ( $17,8 \mathrm{p}$. Ioo). Cela est certainement dû au fait que nous avons étudié des agneaux très jeunes et non castrés. En effet, d'après les résultats comparatifs présentés au tableau 7 , il est clair qu'au cours d'une même période de croissance (entre 15 et $35 \mathrm{~kg}$ ), le gain de poids de tels animaux comporte en moyenne moins de matières grasses (ANDREws et OrSKov, I970) ou de dépôts adipeux (FourIe et al., r970) que celui des mâles castrés (BuRton et Reid, I969; Searle et Mc Graham, I972; Kirton et al., I974; Drew et REID, I975) ou des femelles (RATTRAY et al., I974). En conséquence, la valeur calorifique moyenne du gain de poids vif vide est également plus faible $(2579 \mathrm{cal} / \mathrm{g}$ ) chez les agneaux mâles entiers que chez les animaux castrés (3 300 à $4500 \mathrm{cal} / \mathrm{g}$ d'après les auteurs cités plus haut).

Cette comparaison en terme de composition moyenne du gain de poids ne rend pas compte de l'évolution de la valeur calorifique du croît avec le poids vif vide; c'est pourquoi nous avons calculé la relation entre cette valeur calorifique $(\Delta \mathrm{E} / \Delta \mathrm{P})$ et le poids vif vide $(\mathrm{P})$ en dérivant par rapport à $\mathrm{P}$, l'équation d'allométrie de la valeur énergétique du corps vide :

$$
\text { (I) } \Delta \mathrm{E} / \Delta \mathrm{P}(\mathrm{Mcal})=\mathrm{I}, \mathrm{I} 67 \mathrm{P}^{\mathrm{0}, 261}
$$

Nous avons alors comparé cette équation, à la relation similaire obtenue à partir des résultats de REID et al. (Ig68), relatifs à des agneaux mâles castrés dont le poids vif vide était compris entre I2 et $67 \mathrm{~kg}$.

$$
\text { (2) } \quad \Delta \mathrm{E} / \Delta \mathrm{P}(\mathrm{Mcal})=0,5898 \quad \mathrm{P}^{0,584}
$$

La différence entre les résultats donnés par les deux équations augmente avec le poids vif vide. Elle est égale à $842 \mathrm{kcal} / \mathrm{kg}$ pour un poids vif vide égal à $20 \mathrm{~kg}$ (2 550 contre $3392 \mathrm{kcal} / \mathrm{kg}$ ) et à I I6I kcal $/ \mathrm{kg}$ pour un poids vif vide égal à $25 \mathrm{~kg}$ $(2703$ contre $3864 \mathrm{kcal} / \mathrm{kg})$. De nouveaux résultats, en cours de dépouillement, obtenus avec des agneaux mâles entiers, nous ont montré que la valeur calorifique du gain de poids augmentait très rapidement à partir du poids vif vide de $25 \mathrm{~kg}$. Ceci implique que la différence dans la composition du gain entre agneaux mâles entiers et castrés est certainement plus faible à $25 \mathrm{~kg}$ que celle que nous avons observée. De plus, nous avons utilisé des agneaux alimentés (avant sevrage), au lait reconstitué. Or, Molenat et Theriez (I973) ont observé que le mode d'allaitement (maternel ou artificiel) pouvait modifier de manière hautement significative la teneur en eau à l'âge de 18 semaines de certains dépôts adipeux de couverture, donc la valeur calorifique de ces tissus. Nous avons signalé que la vitesse de croissance globale des animaux avait été relativement faible $(240 \mathrm{~g} / \mathrm{j})$; ceci peut avoir eu une influence sur le gain de matières grasses. Il est donc probable que la valeur calorifique du gain de poids que nous avons observée correspond à une valeur minimale. Ces réserves étant faites, il apparaît cependant que la valeur calorifique du gain de poids, et par conséquent les besoins en énergie nette des agneaux mâles entiers sont plus faibles (de $800 \mathrm{kcal} / \mathrm{kg}$ de gain environ) que ceux des animaux castrés.

Par comparaison aux résultats relatifs aux animaux d'autres espèces, l'augmentation relative de contenu en matjères grasses de nos agneaux ( $b$ voisin de $\mathbf{r}, 5$ ) est du même ordre de grandeur que celle que nous avons obtenve sur des bovins 
mâles entiers (résultats non publiés) ou que les valeurs obtenues sur des porcs (REID et al., I968; Doornenbal, I972). En revanche, elle semble plus lente que chez la souris ( $b=\mathrm{I}, 99$; BAILEy et $a l$., I96o) ou le lapin ( $b=\mathrm{I}, 86$; Vigneron et al., I97I).

Nos résultats confirment l'existence de relations étroites entre les composants chimiques des animaux, décrites par Tulloh (I963), Reid et al. (I968) et LohmaN (I97I) essentiellement; la composition du corps vide est fortement liée $\left(R^{2}>0,99\right)$ au poids vif vide; ce sont les matières grasses qui ont la plus forte variabilité résiduelle, mais leur poids est relié au poids d'eau du corps vide. Cette relation est variable avec l'âge des animaux, car le pourcentage d'eau dans la masse délipidée (poids vif vide — poids des matières grasses) diminue avec l'âge (de 78,8 à 75,2 p. Ioo entre I et I 6 semaines). Cependant, on peut estimer le poids des matières grasses à partir du poids vif vide et du poids d'eau du corps vide avec une erreur résiduelle égale à 6,9 p. Ioo du poids de gras. Il serait donc bien possible d'estimer la composition des animaux vivants à partir de leur poids vif et de leur volume hydrique mesuré indirectement (RoBELIN, I973), à condition que les variations du contenu digestif et de l'eau de ce contenu restent faibles.

Rȩ̣u pour publication en septembre 1976.

\section{Summary}

\section{Changes in the chemical composition of male lambs from $\mathrm{I}$ to $\mathrm{I} 6$ weeks}

Changes in the chemical composition of male lambs was studied by slaughtering four groups of animals aged I, 5, Io and I 6 weeks and weighing $4.8, I 2.4,23.5$ and $32.9 \mathrm{~kg}$, respectively.

During the whole experiment, the percentage of water in empty body decreased from 73.4 to 64.5 per cent, whereas the percentage of fat increased from 6.3 to $\mathrm{I} 4$. I per cent and the calorific value from $I 428$ to $2230 \mathrm{kcal} / \mathrm{kg}$. The percentage of protein ranged around $\mathrm{I} 6.5$ per cent.

The period studied was divided into three sub-periods: I to 5 weeks (milk-fed lambs), 5 to Io weeks (weaning period) and Io to I 6 weeks (fattening period). Relative increase in fat content was higher during the first period (allometric coefficient $b=1.69$ ) than during the second one $(b=\mathrm{I.22})$. It was increasing after weaning $(b=\mathrm{I.4I})$. Relative increase in protein content showed the same variations, but in the reverse order. Changes in fat, protein and energy composition of live weight gain were discussed in relation to feed and physiological state of the animals (puberty).

Comparing with results of the literature, it appeared that the calorific value of life weight gain of entire male lambs during the fattening period was lower $(800 \mathrm{kcal} / \mathrm{kg})$ than that of castrated animals.

\section{Références bibliographiques}

Almguist C. N., Brungardt V. H., Tyler W. J., Waldan R. C., I971. Growth and efficiency of Holstein steers as influenced by live weight and energy intake. J. Dairy Sci., 54, 68I-687.

ANdrews R. P., ORskov E. R., I970. The nutrition of early weaned lamb. II. The effect of dietary protein concentration, feeding level and sex on body composition at two live weights. J. Agric. Sci., 75, 19-26.

Arnal M., Ferrara M., Fauconneau G., 1976. Synthèse protéique in vivo pendant le développement de quelques muscles de l'agneau in "International Symposium on Nuclear Techniques in Animal Production and Health as related to the soil-plant system ". Vienne 1976. 
BaIley C. B., KItts W. D., Wood A. J., 1960. Changes in the gross chemical composition of the mouse during growth in relation to the physiological age. Can J. anim. Sci., 40, I43-I55.

Benevent M., I97I. Croissance relative pondérale postnatale, dans les deux sexes des principaux tissus et organes de l'agneau Mérinos d'Arles. Ann. Biol. anim. Bioch. Biophys., 11, 5-39.

Boccard R., Dumont B. L., 1973. Etude de la production de viande chez les ovins. IX. Variation de l'organisation de la musculature de l'agneau en fonction de la vitesse de croissance. Ann. Zootech., 22, 423-43r.

Burton J. H., REID J. T., I 969 . Interrelationships among energy input body size, age and body composition of sheep. J. Nutr., 97, 517-24.

Doornenbal H., 1972. Growth, development and chemical composition of the pig. II. Fatty tissue and chemical fat. Growth, 36, I85-194.

Drew K. R., REID J. T., I975. Compensatory growth in immature sheep. I. The effect of weight loss and realimentation on the whole body composition. J. Agric. Sci., 85, 193-204.

Fourie P. D., Kirton A. H., Jury K. E., I970. Growth and development of sheep. II. The effect of breed and sex cn the growth and carcass composition of the Sowthdown and Romney and their cross. N.Z. J. Agric. Res., 13, 753-770.

Jagusch K. T., Norton B. W., Walker D. M., r97o. Body composition studies with the milk fed lamb. II. The effect of the age of the lamb and the protein content of the diet on the chemical composition of the body and its organs. J. Agric. Sci., 75, 279-285.

KIrton A. H., Fourie P. D., JURy K. E., 1972. Growth and development of sheep. III. Growth of the carcass and non-carcass components of the Sowthdown and Romney and their cross, and some relationships with composition. N.Z.J. Agric. Res., 15, $214-27$.

Kirton A. H., Dalton D. C., Ackerley I. R., I974. Performance of sheep on New Zealand hill country. N.Z.J. Agric. Res., 17, 283-93.

Lohman T. G., I97I. Biological variation in body composition. J. Anim. Sci., 32, 647-653.

Lohse C. L., Moss F. P., Butterfield R. M., 197I. Growth patterns of muscles of Merino sheep from birth to 517 days. Anim. Prod., 13, I I7-1 26.

Molenat G., Theriez M., Aguer D., I97I. L'allaitement artificiel des agneaux. I. Détermination de l'âge minimal au sevrage pour la production d'agneaux de boucherie. Ann. Zootech., 20, 339-352.

Molenat G., Theriez M., I973. Influence du mode d'élevage sur la qualité de carcasse de l'agneau de bergerie. Ann. Zootech., 22, 279-293.

Ratrray P. V., Garrett W. N., Hinman N., East N. E., 1974. Effect of level of nutrition, pregnancy and age on the composition of the whole free ingesta-free body and carcass of sheep. J. Anim. Sci., 39, 687-693.

Reid J. T., Bensadoun A., Bull L. S., Burton J. H., Gleeson P. A., Han I. K., Joo Y. D., Johnson D. E., McManus W. R., Paladines O. L., Stroud J. W., Tyrrell H. F., Van NiEkeRK B. D. H., Wellington G. H., Wood J. D., I968. Changes in body composition and meat characteristics accompanying growth of animals. Proc. Cornell Conf. Feed. Manuf., I8-37.

Robelin et Geay, 1976. Estimation de la composition des carcasses de jeunes bovins à partir de la composition d'un morceau monocostal prélevé au niveau de la i I $\mathbf{e}$ côte. II. Composition chimique. Ann. Zootech., 25, 259-272.

Rouse G. H., Topel D. G., Vetter R. L., Rust R. E., 197o. Carcass composition of lambs at different stages of development. J. Anim. Sci., 31, 846-854.

Robelin J., 1973. Revue bibliographique : Estimation de la composition corporelle des animaux à partir des espaces de diffusion de l'eau marquée. Ann. Biol. anim. Bioch, Biophys., 13, 285-305.

Schultz E., Oslage H. J., Daenicke R., I974. Untersuchungen über die zusammensetzung der korpersubstanz sowie den stoff-und energieansatz bei wachsenden mastbullen. Z. Tierphysiol., Tiernährg. Futtermittelkde. Beheifte no 4 .

Searle T. W., Grahman N. M. C., O'Callaghan M., I972. Growth in sheep. I. The chemical composition of the body. J. Agric. Sci., 79, 371-382.

Tullow N. M., I963. The carcass composition of sheep, cattle and pigs as function of body weight. In "Carcass composition and appraisal of meat animal". 5, I-16. Ed. D. E. Tribe. East Melbourne. C.S.I.R.O.

Vigneron P., Baron R., Dauzier L., i97I. Evolution postnatale de la quantité d'eau et de lipides du corps et du grand psoas chez le Lapin. Ann. Biol. anim. Bioch. Biophys., 11, 669679 . 